
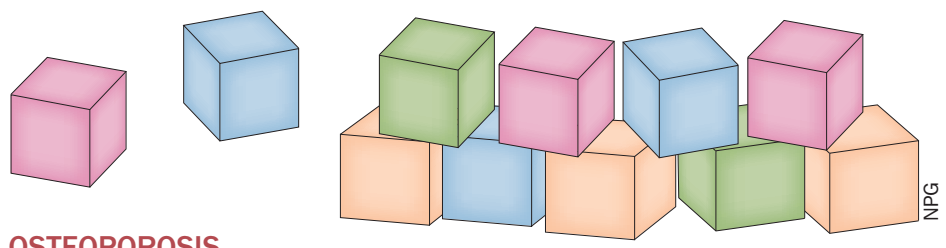

\title{
OSTEOPOROSIS
}

\section{Novel antibody therapy shows potential for building bone}

Targeting a naturally occurring inhibitor of bone formation could signal a new direction for osteoporosis care. Findings from an international phase II clinical trial reported in the New England Journal of Medicine indicate that administering romosozumab-a humanized monoclonal antibody directed against sclerostinincreases bone mineral density (BMD) among individuals with low bone mass.

Sclerostin is an osteocyte-derived glycoprotein that acts through the Wnt and bone morphogenetic protein signalling pathways to inhibit osteoblast function; consequently, bone formation is decreased. As expression of sclerostin is restricted to the skeleton, inhibition of this factor represents an ideal candidate for targeted therapeutic intervention.

In the phase II randomized controlled trial, conducted by researchers from 28 centres, the primary outcome measure was the percentage change from baseline in BMD at the lumbar spine. Secondary end points included percentage changes in BMD at other anatomical sites and in biochemical markers of bone turnover.

The study group evaluated the effects of five different dosing regimens of romosozumab (administered by subcutaneous injection) over a 12-month period among 419 postmenopausal women (mean age 67 years) with osteopaenia or osteoporosis. Responses to romosozumab were weighed against those observed with placebo injections and two active comparators, alendronate and teriparatide.

The researchers found that all doses of romosozumab markedly increased BMD in the spine and hip. Notably, with the largest dose of romosozumab $(210 \mathrm{mg}$ administered each month), the increase in lumbar spine BMD at 12 months (11.3\%) was significantly greater than the response to either alendronate $(4.1 \%)$ or teriparatide (7.1\%). By contrast, participants assigned to the placebo group experienced a $0.1 \%$ decrease in BMD at the lumbar spine during this timeframe.

Biochemical indices of bone formation increased during the first 6 months of romosozumab administration but then dropped back to baseline levels thereafter, whereas levels of a bone resorption marker were modestly decreased from baseline during the full 12 months of romosozumab therapy.
Injection-site reactions occurred more frequently among the groups receiving romosozumab than the placebo group; however, these effects were generally mild and did not exhibit a dose-response relationship. The incidence of serious adverse effects was $7 \%$ with romosozumab and $14 \%$ with placebo.

The novelty of the study findings for driving future targeted treatment of osteoporosis is confirmed by Carolyn Becker (Brigham and Woman's Hospital, Boston, USA), an expert in bone disease. Writing in an accompanying editorial, Becker states "The pattern of brief anabolic stimulation coupled with chronic suppression of bone resorption seen with romosozumab is unprecedented among current therapies for osteoporosis."

\section{Vicky Heath}

This article is modified from the original in Nat. Rev. Endocrinol. (doi:10.1038/ nrendo.2014.1).

Original article McClung M. R. et al. Romosozumab in postmenopausal women with low bone mineral density. N. Engl. J. Med. doi:10.1056/NEJMoa1305224 